\title{
La voz de las personas en situación de vulnerabilidad en el diseño de las políticas sociales en Euskadi. La experiencia de EAPN Euskadi
}

\author{
M. Elena de la Hera Rodríguez \\ EAPN Euskadi \\ <tecnico@eapneuskadi.net>
}

\begin{abstract}
EAPN Euskadi sarea osatzen dute gizarte-kaltebera egoeran dauden biztanleekin lan egiten duten erakunde sozial batzuek, eta xede dute pobreziaren aurkako eztabaida soziopolitikoa sustatzea.

Oinarritzat hartzen dugu pobrezia eta gizartebazterketa ezabatu ahal izateko politika sozial eta ekimenetan pertsona horien iritzia barneratzea. Izan ere, biztanle horien parte-hartzearen gabezia berdintasun ezaren arrailetako bat baita, oinarrizko eskubide, ezagutza, gutxiagotasunaren sentimendu eta gaitasunen gabeziarekin bat. Gure asmoa da Euskadiko politika sozialak diseinatzerakoan pertsona horien iritzia kontuan hartzea, modu honetan eragin eta kezkatzen dituzten gizarteratzeko sistemaren baitan dauden alderdien inguruan berauen esana entzun ahal izateko.
\end{abstract}

\section{GAKO-HITZAK:}

Parte-hartzea, gizarteratzea, gizarte-politika, eragina, pobrezia, bazterkeria.
EAPN Euskadi es una red que aglutina entidades sociales que trabajan con personas en situación de vulnerabilidad para propiciar el debate sociopolítico en torno a la lucha contra la pobreza. Partimos de considerar que la opinión de estas personas es clave en la propuesta de políticas sociales y acciones para erradicar la pobreza y la exclusión social. La participación de estas personas es una más de las brechas de desigualdad que existen, junto con el acceso a derechos fundamentales, el conocimiento, el sentimiento de inferioridad y las aptitudes. Pretendemos promover que la opinión de estas personas sea tenida en cuenta a la hora de diseñar políticas sociales en Euskadi, dándoles voz sobre los aspectos relacionados con el sistema de inclusión social que les afectan y les preocupan.

\section{PALABRAS CLAVE:}

Participación, inclusión social, política social, incidencia, pobreza, exclusión. 


\section{Introducción}

La participación de las personas en situación o riesgo de pobreza y exclusión social que son atendidas en entidades sociales (servicios de atención social) puede ser entendida desde diferentes perspectivas. De cómo se entienda en las organizaciones la participación de las personas que la componen, y especialmente de las personas atendidas, va a depender el rol que se les asigne dentro de su propio proceso de inclusión y, por tanto, si se les tiene en cuenta o no dentro del diseño de las políticas sociales.

En la Red Europea de Lucha contra la Pobreza y la Exclusión Social (EAPN 1 ), se apuesta de forma clara por que "las personas puedan proponer y evaluar las políticas que les afectan directamente” (Susías, 2012: 9-10), contribuyendo, de esta manera, a potenciar una ciudadanía activa, una ciudadanía que se implique en aquellos aspectos que los influyen de manera tanto individual como colectiva.

En 2005, diferentes entidades que trabajaban con personas en situación de pobreza y exclusión social y que formaban parte de la Red de Lucha contra la Pobreza y la Exclusión Social en Euskadi (EAPN Euskadi) constituyeron un grupo de trabajo con el objetivo de promocionar la participación de las personas a las que estaban atendiendo dentro de las propias entidades, personas que también son parte de las entidades y que, por tanto, deben formar parte de los procesos de toma de decisiones de los servicios en los que son atendidas. Se constituía, así, el germen del compromiso que EAPN Euskadi ha adquirido y renovado en los últimos doce años de defender la necesidad de tener en cuenta a las personas que sufren o han sufrido pobreza y exclusión social en el diseño de las políticas de lucha contra la pobreza de la Comunidad Autónoma del País Vasco (CAPV).

\section{Inclusión social, participación...: de la práctica diaria a la construcción de la teoría}

El trabajo de la Red en el ámbito de la participación de las personas que experimentan o han experimentado pobreza y exclusión social es fruto de años de reflexión conjunta tanto con profesionales como con personas atendidas en servicios de las entidades sociales que forman la Red. Partimos, así, del trabajo diario de los servicios de atención a personas en situación o riesgo de pobreza y exclusión social, y de los conceptos clave en el ámbito de la inclusión social y la participación, para materializar una teoría propia construida a partir de la práctica diaria en estos servicios.

${ }^{1}$ Las siglas corresponden al nombre de la red en inglés: European anti Poverty Network.
A este respecto, la Guía metodológica de la participación social de las personas en situación de pobreza y exclusión social editada por la red estatal en 2010 es fundamental para comenzar nuestra reflexión. No nos extenderemos aquí en definir y teorizar conceptos como pobreza, exclusión social o inclusión, pero sí creemos necesario hablar sobre lo que en la Red entendemos por participación.

Una de las primeras tareas que se propone este grupo de trabajo, que aglutina a profesionales de diferentes entidades sociales en Euskadi, fue revisar diferentes definiciones de participación, desde sus diferentes vertientes, siempre con el fin de poder crear un lenguaje común. Éste es un aspecto especialmente relevante, ya que, como decíamos en la Guía... (EAPN España, 2010: 24), “de cómo entiendan la participación las propias organizaciones sociales, dependerán los procesos que se pongan o no en marcha para promoverla".

Dentro de las propias organizaciones, se puede tener en cuenta la participación de los diferentes grupos de personas que las engloban:

- Tenemos, por un lado, la base social de la propia entidad (incluiríamos aquí a los órganos de dirección propios de cada entidad: asambleas, juntas directivas, patronatos).

- Por otro lado, podemos hablar también de la participación de las personas que forman parte de las entidades por estar involucradas en su gestión diaria o en la atención a las personas que utilizan los servicios. Incluimos aquí a todas las personas profesionales, equipos de gestión y voluntariado de atención a personas o que realiza tareas diversas de apoyo a la atención directa.

- Y tenemos, además, la participación de las personas que son atendidas dentro de las entidades sociales y sus servicios, dentro de diversos procesos de inclusión social. Un grupo que, muchas veces, se nos olvida al hablar de la participación.

En EAPN Euskadi, elegimos trabajar precisamente la participación de las personas en situación o riesgo de pobreza y exclusión social. Aquellas personas con las que directamente se compromete la Red desde su creación, ya que su fin último es la de aglutinar a entidades sociales para, entre otras cosas, "constituir un grupo de presión con capacidad de interlocución, informando y denunciando las situaciones de pobreza y exclusión, propiciando el debate sociopolítico y presentando alternativas válidas para superar esas situaciones" (misión de EAPN Euskadi²). Tiene, por tanto, lógica que una de las cuestiones que tomemos en consideración sea, precisamente, la voz de las personas que se encuentran en ese tipo de situaciones o las hayan vivido.

2 Véase 〈http://www.eapneuskadi.org/es-eapn_euskadi_presentacion〉. 


\subsection{La participación como proceso}

En toda esta reflexión teórico-práctica que emprendemos sobre la participación, nunca debemos perder de vista cuál es el objetivo de la Red y de los servicios de las entidades sociales que la componen, que no es otro que la lucha contra la pobreza y la exclusión social, es decir, proponer cauces y soluciones para lograr la inclusión social de las personas que se encuentran en situación de vulnerabilidad social. No se trata de la participación por la participación, sino de establecer cauces para que las personas en estas situaciones contribuyan, con su voz y experiencia, a la lucha contra la pobreza en Euskadi.

Teniendo esto en cuenta, dentro de las organizaciones podemos tomar la participación como un proceso o como un medio para conseguir el objetivo último que nos proponemos. Ambas vertientes son válidas siempre y cuando tengamos claro lo que implica cada una de ellas y sus limitaciones. A este respecto, podemos decir que muchas veces la participación de las personas atendidas y el conocimiento sobre participación se circunscribe a técnicas que se utilizan en determinadas actividades en las que se piden opiniones o valoraciones sobre aspectos concretos. Por supuesto, se trata de un comienzo, pero no alcanza, en la mayoría de los casos al resto de la organización ni se convierte en algo estratégico (reflexionado, pensado, con un fin último y aceptado dentro de toda la organización).

Este tipo de acciones, si se organizan bien, pueden tener muy buenos resultados. Si seguimos reflexionando en esta línea y somos consciente de cómo hacemos las cosas en las organizaciones, podemos lograr que la participación se convierta en un proceso, y de este modo, en algo estratégico, como veremos más adelante: "la participación como proceso hace referencia a las 'personas', mientras que la participación como medio hace referencia a las 'cosas'. Cosas como actividades, protocolos, procedimientos, programas..." (EAPN España, 2010: 25). La participación como proceso deja atrás la visión instrumental; ya no se trata de colaborar, sino que participar implica ser parte, $y$ todo proceso para ser parte de algo implica poderse comunicar entre unos y otros, rompiendo barreras y jerarquías que muchas veces establecemos, de manera inconsciente, en las organizaciones en el propio proceso de intervención social. Implica tener conciencia de qué estamos haciendo en cada servicio y entidad con respecto a la participación de las personas y, lo que es más importante, por qué.

Se han elaborado diferentes modelos teóricos para explicar y caracterizar los niveles de participación de la población y de grupos sociales determinados. Nos parece muy interesante la descripción que se hace al respecto en el cuaderno conceptual elaborado para la caja de herramientas editada por EAPN España bajo el título de Crea, juega y participa. Caja de herramientas para la participación; un trabajo en el que se tuvieron en cuenta también las reflexiones de los grupos de participación de las EAPN territoriales. En esta obra, se define la participación como una escalera en la que cada escalón representa un nivel diferente de participación e implicación ${ }^{3}$ :

- Escalón 1: participación manipulada. [...] La población es utilizada para realizar acciones que no entiende y responde totalmente a intereses ajenos a los suyos.

- Escalón 2: participación decorativa. Se realiza cuando se incorpora a la población como un accesorio, es decir, 'para decorar' o 'animar' determinada actividad. Asimismo, cuando en determinadas publicaciones o acciones es incorporada sólo con fines decorativos o 'folklóricos'.

- Escalón 3: participación simbólica. Es la que podemos apreciar cuando se realizan acciones donde la participación de la población es sólo aparente.

- Escalón 4: participación de asignados, pero informados. En este nivel, aún se dispone de la población para que participe en una determinada actividad, sin embargo, se le informa [de] en qué consiste la misma. Es, aunque todavía limitada, el primer nivel de participación real.

- Escalón 5: participación con información y consulta. Es el segundo nivel de participación real. En él, los agentes externos informan y consultan a la población sobre su probable participación. En base a ello, la población decide.

- Escalón 6: participación en ideas de agentes externos de desarrollo compartidas con la población. La acción es pensada por agentes externos, pero es compartida con la población. Supone que ellos y ellas se incorporan en pensar y aportar respecto a la acción a realizar.

- Escalón 7: participación en acciones pensadas y ejecutadas por la propia población. La acción se gesta en la propia población y es ejecutada con ellos y ellas. No hay relación con agentes externos. Sin duda, son las personas las protagonistas de la acción. Sin embargo, no cuentan con el aporte que podrían brindar la experiencia o dominio técnico de los agentes externos.

- Escalón 8: participación en acciones pensadas por la propia población y que han sido compartidas con agentes externos de desarrollo. La acción es pensada por la población, pero a diferencia del escalón anterior, es compartida con agentes externos. Es el nivel superior que contempla la llamada escalera de la participación (Carrasquilla y Carnacea, 2013: 32-33).

En los tres primeros escalones, no hay una verdadera participación de las personas implicadas. ¿Serían los escalones siguientes a los que deberíamos aspirar en todos los casos? Sin duda, la participación ideal

${ }^{3}$ Este modelo se basa en un artículo de Sherry Artein (1969). 
es la que parte de la propia ciudadanía, pero hemos de tener en cuenta que trabajamos con colectivos que no siempre tienen acceso a estos últimos niveles, dadas sus situaciones concretas, y no debemos tampoco olvidar el objetivo específico de cada entidad o servicio. Por tanto, no sería nuestra meta llegar al último escalón, el nivel ideal de la participación, sino situarnos como organizaciones sociales, con coherencia, en el que nos corresponda en cada caso, siempre dentro de los niveles reales de participación y sin manipular a las personas (siendo conscientes de las limitaciones y de las ventajas en cada caso y haciendo, asimismo, conscientes de ello a todas las personas implicadas).

\subsection{Nuestra práctica, nuestra teoría}

Teniendo en cuenta todas estas cuestiones, y conscientes de los diferentes niveles de participación que se podían manejar y lo que cada nivel conlleva, en 2009 y en el marco del trabajo del Grupo de Participación de EAPN Euskadi, se decide dar un paso más en el análisis de la participación de las personas atendidas dentro de las propias organizaciones, intentando recoger también la opinión de las personas sobre las técnicas que utilizamos para potenciar la participación.

Los perfiles de las personas con las que intervienen estas entidades son muy variados, aunque todos tengan en común una situación de vulnerabilidad o riesgo de exclusión que les hace difícil participar en cualquier proceso, individual o colectivo, que favorezca su inclusión social. Son personas que, una vez han accedido a una entidad social, entran en un proceso de inclusión en función de las necesidades y demandas de cada una, con un seguimiento realizado por profesionales de la entidad social. Durante los últimos años, se había notado que las personas atendidas participaban de las organizaciones solamente en momentos puntuales (recordemos los escalones de nuestra escalera de participación, de la que hemos hablado), ya fuera en la organización de actividades o acciones, ya fuera en su propio proceso de inclusión. Esta carencia de participación se hacía más evidente por las condiciones de grave exclusión y vulnerabilidad en las que se encontraban muchas de estas personas. Sin embargo, se daba la paradoja de que, generalmente, las entidades sociales comenzaron a trabajar en los barrios, y en estos comienzos, la participación era muy alta. Es decir, a pesar de que las entidades sociales de nuestro territorio tienen una historia relativamente reciente (30-40 años en su mayoría), en el proceso necesario de profesionalización habían perdido esa vertiente de participación, podemos llamar, comunitaria; participación que, por otro lado, fue lo que contribuyó a su puesta en marcha.

Considerando la historia de nuestras propias entidades, pero sin centrarnos en ella, el Grupo de Participación de EAPN Euskadi ya había realizado un análisis de las herramientas y técnicas que las propias entidades y servicios estaban utilizando para conocer las necesidades y los niveles de participación de las personas con las que trabajaban. Pero este primer análisis se realizó desde el punto de vista de las personas profesionales. Así, en una segunda fase se planteó la realización de una experiencia piloto, con el objetivo de dar voz a las personas atendidas en los diferentes servicios y, de este modo, conocer sus valoraciones sobre las herramientas que se estaban utilizando en las entidades.

Los aspectos más innovadores de la experiencia que se llevó a cabo fueron dos, porque el seguimiento y la sistematización de las diferentes experiencias se centralizaban en la propia Red:

- Se trataba de entidades diferentes (en todos sus aspectos: ideario, tipología de personas atendidas y de servicios prestados, número de personas trabajadoras o presupuesto anual manejado), lo que nos permitió conocer si se podían obtener conclusiones parecidas de cada experiencia y nos podía facilitar la tarea de transferencia del proceso. Asimismo, era necesario utilizar técnicas diferentes para cada entidad.

- Por otro lado, el grupo partía desde la práctica, es decir, desde lo que de hecho se estaba realizando, para evaluarlo y sacar conclusiones que se convertirían en la teoría.

Esta experiencia piloto fue plasmada en el documento La participación como herramienta para la inclusión social. Experiencias piloto en entidades sociales de Euskadi para el fomento de la participación social, editado por EAPN Euskadi en 2011. Asimismo, podemos señalar que esta experiencia fue seleccionada como buena práctica a escala europea en materia de participación social de personas en situación de vulnerabilidad en el documento sobre experiencias locales de participación de EAPN Europa (2012).

Como resumen de la experiencia, podemos mencionar algunas de las conclusiones que obtuvimos. Todas ellas se han tenido en cuenta en los siguientes procesos de participación desarrollados por la Red:

- Sobre el enfoque ideológico de la participación:

- La propia sociedad en la que vivimos es excluyente, y también lo es a la hora de proponer cauces de participación.

- Debemos generar conciencia crítica, y para ello es necesario no centrarnos solamente en el proceso individual de cada persona, aunque sea el aspecto más importante dentro del trabajo que realizamos en el proceso de inclusión social.

- La participación es transformadora, y por tanto, debe ser un proceso colectivo y no debemos quedarnos en la situación individual.

- La participación debe servir para reducir las diferencias y la brecha de desigualdad. 
- Sobre la apertura de procesos de participación dentro de las entidades sociales:

- Debemos entender el proceso de inclusión como un proceso de autonomía, y desarrollar procesos (y cauces) de participación. La participación debe ser considerada como una herramienta importante en la inclusión social.

- Contemplar la participación como herramienta para la inclusión social implica que cada entidad debería entender la participación de las personas atendidas como un reto propio y convertirlo en una de sus líneas estratégicas.

- Esto quiere decir que la participación debería ser un proceso transversal y no debería tratarse como un aspecto más (un apartado de otros, con su espacio 'especial').

- Sobre las herramientas y procedimientos que se usan para fomentar la participación de las personas:

- Es necesario tomarse tiempo y priorizar el análisis de lo que ya estamos desarrollando en clave de participación. Es decir, sistematizar las experiencias de cara a identificar áreas de mejora o profundizar en aspectos que ya se están trabajando, antes de crear nuevos espacios o herramientas. Analizar y evaluar para mejorar.

- Debemos tener en cuenta que, aunque las herramientas o cauces de participación existan, no está garantizada la participación de las personas. Es preciso conocer, dar a conocer y establecer una dinámica y una estrategia de trabajo en torno al fomento de la participación.

- Es necesario adecuar los espacios y el lenguaje.

- Sobre las personas que forman parte de las entidades sociales y están implicadas en los diferentes procesos:

- Cada persona dentro de una entidad juega un rol diferenciado. Debemos conocer estos roles y conocer la realidad de nuestra entidad, así como cuáles son los miedos y dificultades. Esto nos permitirá establecer una estrategia para afrontar las dificultades y vencer resistencias. Este proceso debe desarrollarse con todas las personas que conforman la entidad, y nos ayudará a aprender a adaptarnos.

- Promover la participación significa crear espacios compartidos y romper roles establecidos. Debemos plantearnos y respondernos hasta dónde estamos dispuestos a llegar, hasta dónde estamos dispuestos a cambiar lo establecido. En función de nuestra respuesta, desarrollaremos una estrategia más realista.

- Una de las máximas que debemos adoptar es que debemos dar voz a las personas, y no hablar por ellas.
- Sobre la influencia del entorno y sus potencialidades:

- Debemos plantearnos las siguientes preguntas en cuanto al entorno de nuestra entidad: ¿lo conocemos?, ¿qué ventajas nos ofrece?, ¿en qué nos puede perjudicar?

- Es importante conocer el entorno de las personas con las que trabajamos y cómo es su participación en él. Cada persona adquiere un rol diferenciado en cada uno de los espacios donde se relaciona; es decir, que una persona no participe en la entidad no tiene por qué significar que no participe en otros espacios (hay tener en cuenta los intereses de las personas, y no dar nada por sabido o conocido).

\section{La participación de las personas en situación de vulnerabilidad como estrategia dentro de las organizaciones}

En 2014, EAPN Euskadi incorpora como línea prioritaria estratégica la recogida de opinión de las personas atendidas en las entidades que integran la Red sobre cuestiones relacionadas con la lucha contra la pobreza y la exclusión social, y sobre las políticas sociales en Euskadi. Tal y como especifica el propio Plan Estratégico 2015-2017, EAPN Euskadi mantiene y renueva su compromiso con las personas en situación de vulnerabilidad, y concreta ese compromiso en esta línea estratégica, que tiene como finalidad la autonomía, la defensa, el apoyo, la promoción y el empoderamiento de las personas en situación de vulnerabilidad. Con ello, se intenta propiciar que las personas en situación de vulnerabilidad y exclusión puedan ejercer o hacer efectivos sus derechos y deberes como cualquier ciudadano o ciudadana (con el mismo nivel de oportunidades, con la misma información, con los mismos recursos); que puedan acceder a servicios y recursos de calidad (que los servicios públicos dirigidos a toda la ciudadanía sean accesibles para ellas, y que los especializados y específicos tengan unos niveles de calidad adecuados, que respeten su dignidad) y que tengan espacios para promocionar su participación en todos los ámbitos de su vida. Es por ello por lo que se habilitan los espacios necesarios para recoger esas opiniones, siempre dentro de una estrategia de trabajo.

A este respecto, y teniendo en cuenta nuestros planteamientos teórico-prácticos, las reflexiones y los trabajos previos realizados, en EAPN Euskadi defendemos la importancia de fomentar la participación dentro de las organizaciones sociales. En primer lugar, por ser un entorno conocido y en segundo, porque es en esta participación donde las personas atendidas sienten que realmente aportan (se trata de una realidad inmediata que les afecta de forma inmediata).

Empezar a recoger la opinión de las personas sobre los servicios que las atienden supone tenerlas en 
cuenta en los procesos de toma de decisiones. Como señalábamos en el apartado anterior, es importante señalar que iniciar procesos para fomentar la participación dentro de las organizaciones sociales debe ser producto de una reflexión que se inicie dentro de la propia entidad, no algo impuesto desde fuera. La entidad debe asumir el inicio del proceso de participación de personas usuarias o atendidas como un reto propio, y convertirlo en línea estratégica de la organización. Asimismo, la experiencia de trabajo nos dice que, para ser sostenible en el tiempo, la participación y los procesos de participación deben ser transversales dentro de las organizaciones; su inicio debe ir acompañado de una reflexión que lleva tiempo y recursos; reflexión en la cual habrá que priorizar aspectos como la formación y el cambio de actitudes respecto a cómo estamos trabajando e interviniendo en procesos de inclusión social. Por todo ello, la entidad debe tener muy claras las implicaciones de esta estrategia y la posibilidad de realizar cambios organizacionales coherentes una vez se inicien procesos de participación en los que se incluyan a las personas atendidas en los servicios.

Además, cuando se inician estos procesos dentro de las entidades sociales, es necesario trabajar con todas las personas que forman parte de la organización. Esto implica que deben analizarse aspectos como los roles de todas las personas que participan dentro de la organización, la dificultad de romper con roles ya establecidos, o lo que puede suponer para los y las profesionales (y para las propias personas atendidas) romper esos roles. Abrir este proceso puede significar introducir cambios importantes en lo que ya está establecido, y puede provocar recelos y crisis dentro de la propia organización. Debemos conocer y analizar nuestra propia organización, y establecer límites coherentes a su ideario, sin perder nunca de vista el objetivo último de los servicios que tenemos en marcha. Como decíamos al inicio de este artículo, "de cómo entiendan la participación las propias entidades sociales, dependerán los procesos que se pongan o no en marcha para promoverla" (EAPN España, 2010: 24).

Considerando todas estas premisas en cuanto a las organizaciones que forman parte de la Red Europea de Lucha contra la Pobreza y la Exclusión Social, el respeto hacia todas y cada una de ellas, hacia sus variados idearios y formas de intervenir, EAPN Euskadi ha ido tomando decisiones en cuanto al establecimiento como línea estratégica de la participación de las personas que sufren o han sufrido pobreza y exclusión social.

Respecto a la participación de personas en situación o en riesgo de pobreza y exclusión social, debemos señalar que el camino a recorrer es aún largo. Los espacios de participación en las entidades suelen ser todavía limitados y se circunscriben al funcionamiento diario de la entidad o servicio al que acuden, siendo prácticamente inexistente la representación de estas personas en los órganos de dirección. El conocimiento de las políticas sociales es, por tanto, muy reducido (la mayoría de las veces, se centra en su propia experiencia personal); pero no nos engañemos, pues no por ello resulta inválido. Como profesionales, debemos aprender a extrapolar conclusiones, teniendo en cuenta las diferentes situaciones, aprendiendo a diferenciar y a valorar, por encima de la queja, la propuesta real.

Entendiendo que la participación de las personas es clave en la erradicación de la pobreza y la exclusión social, EAPN Europa plantea tres mensajes clave:

1. Las personas que experimentan pobreza y exclusión social son parte esencial en la solución del problema.

2. Hay que hacer que la estrategia Europa 2020 sea responsable ante las personas, y que éstas puedan acceder al Parlamento Europeo.

3. Debe priorizarse la dimensión social y asegurarse fondos europeos para la lucha contra la pobreza, con inversión social en trabajo de calidad, servicios y protección social.

Por ello, EAPN Euskadi reconoce la necesidad imperativa de apoyarnos en las personas que experimentan o han experimentado pobreza y exclusión social para proponer y construir nuevas políticas sociales o para mejorar las que ya existen. Tener en cuenta la experiencia de estas personas nos permitirá ahondar en las situaciones de pobreza y exclusión social de la CAPV, así como proponer soluciones viables, adecuadas y sostenibles. Una de las cuestiones en las que debemos insistir es en trabajar la participación de las personas en el diseño de políticas sociales, ofreciéndoles espacios donde elevar su voz, pero haciéndolo de manera coherente, teniendo también en cuenta, como explicábamos, los entornos conocidos de las personas, y no creando espacios artificiales, que podrían pervertir las opiniones.

\section{La opinión de las personas sobre las políticas sociales que les afectan}

Como venimos diciendo, EAPN Euskadi se constituye como un grupo de presión con capacidad de interlocución, que informa sobre las situaciones de pobreza y exclusión social y las denuncia, que propicia el debate sociopolítico y presenta alternativas válidas para superar esas situaciones. Dentro del trabajo que realiza en el ámbito de la incidencia en política social, las propias personas que sufren o han sufrido pobreza y exclusión social han sido las grandes olvidadas; muchas veces, ellas mismas se quejan de 'ser invisibles' ante el resto de la sociedad. Citando a Alicia Suso (2012: 72), miembro del grupo de investigación Parte Hartuz de la Universidad del País Vasco / Euskal Herriko Unibertsitatea (UPV/EHU), "hablar de participación sin tener en cuenta las condiciones de vida reales de las personas supone construir un discurso vacío, susceptible de ser adornado con grandes conceptos, que incluso pueden servir para enmascarar la 
complicada situación de estos sectores sociales cada vez más vulnerables y con mayor incertidumbre”.

Desde la reflexión de EAPN Euskadi sobre la participación de las personas que tienen experiencia en pobreza y exclusión social, y la necesidad de tenerlas en cuenta en el debate sociopolítico para que las políticas de lucha contra la pobreza sean efectivas, creemos con firmeza que es necesario “mantener una dinámica tendente a favorecer la participación de las personas en todos los ámbitos de su vida; se trata de que las personas formen parte de su comunidad, tomen parte en las decisiones que les atañen, se oiga su voz, su opinión sea tenida en cuenta” (EAPN Euskadi, 2011: 5).

Por ello, EAPN Euskadi ha habilitado los mecanismos necesarios para que las personas en situación de vulnerabilidad sean cada vez más partícipes tanto en la propia Red como en las entidades y servicios en que los que son atendidas, entendiendo esta participación como algo intrínseco a su proceso de inclusión social y, por tanto, de autonomía. Así, los objetivos que se plantea EAPN Euskadi con respecto a los espacios de participación y de recogida de opinión de las personas que experimentan o han experimentado pobreza y exclusión social son los siguientes:

- Dar voz a las personas atendidas en entidades sociales.

- Obtener propuestas concretas de mejora tanto en los servicios a los que acceden como en las políticas sociales, recogiendo propuestas y recomendaciones sobre asuntos que les afectan en el día a día. Es decir, elevar la voz de las personas en riesgo o situación de exclusión social.

Para poder dar voz a las personas y, asimismo, elevar su voz adonde corresponda, EAPN Euskadi se constituye como altavoz. No se trata de ser la voz de las personas en situación de vulnerabilidad - porque éstas tienen su voz propia, sus opiniones y su manera de pensar-, sino de recoger su opinión y propuestas para llevarlas adonde corresponda, sin medias tintas. Y lo hacemos a través de dos estrategias:

- Facilitando la participación en la entidad o servicio en los que la persona es atendida.

- Mediante los encuentros de participación de personas con experiencia en pobreza y exclusión social que organizamos.

\subsection{La participación en la entidad o servicio en los que la persona es atendida}

Como hemos explicado, EAPN Euskadi apuesta decididamente por el fomento de la participación de las personas dentro de la propia entidad, y toma asimismo en cuenta la importancia de cada una de las personas en sus propios entornos cercanos (familiar, de barrio). Es preciso resaltar el trabajo de la entidad y de los servicios en los que son atendidas las personas, y no ejecutar saltos innecesarios y artificiales en la escalera de la participación. Todos y todas nosotras nos sentimos más cómodas en entornos conocidos, donde nuestras opiniones son más sinceras. Que nuestra opinión se tenga en cuenta en los espacios que frecuentamos, aquellos que son conocidos e inmediatos, nos permite implicarnos y conocer los efectos de nuestra participación. Nos permite ser parte.

Las personas deben ser protagonistas de su propio proceso; por ello, se deben visibilizar los logros particulares de cada una (teniendo en cuenta a la persona y su manera de ser) y los logros de los grupos. Además, es importante darnos cuenta, como decíamos, de que debemos dar voz a las personas y no hablar por ellas. El rol que los y las profesionales debemos tener en todo este proceso de participación que hemos iniciado será el de proponer y adecuar los cauces de participación; que éstos sean conocidos, pero que sean las personas quienes decidan si participan o no. Y cada toma de decisiones, siempre y cuando seamos conscientes y conozcamos todas las posibilidades que tenemos, debe ser considerada participación.

\subsection{Los encuentros de participación de personas con experiencia en pobreza y exclusión social de EAPN Euskadi, un hito en la recogida de la opinión de las personas atendidas sobre políticas sociales y de lucha contra la pobreza en el País Vasco}

La iniciativa nace del Grupo de Participación de EAPN Euskadi. Entendiendo que la participación es una más de las brechas de la desigualdad -junto con el acceso a derechos fundamentales, el conocimiento, el sentimiento de aislamiento y las aptitudes $-y$ considerando lo aprendido en etapas de trabajo anteriores, en EAPN Euskadi decidimos dar otro paso y organizar un acto público en el que reuniéramos a personas a las que afectaban, de primera mano, las políticas sociales que se estaban desarrollando para luchar contra la pobreza y la exclusión social; políticas que se traducen en servicios concretos y en ayudas sociales que se ofrecen a la ciudadanía en situación de pobreza, exclusión social o riesgo, y que, asimismo, regulan el acceso y la tramitación a esos servicios y ayudas ${ }^{4}$.

El primer encuentro de participación de personas en situación de pobreza y exclusión social organizado por EAPN Euskadi responde, además, a dos demandas específicas de las personas con las que trabajamos en las entidades:

${ }^{4}$ Debemos tener en cuenta que las personas atendidas en diferentes servicios de carácter social, así como la ciudadanía en general, no tienen conciencia ni conocen las 'políticas de lucha contra la pobreza'. Para llegar a este concepto, debemos, por tanto, hablar de aspectos conocidos, como los servicios sociales, las ayudas o las prestaciones. 
- Por un lado, personas que participaron en un encuentro estatal de participación en 2013 nos plantearon la necesidad de que nos encontráramos en Euskadi y debatiéramos sobre la realidad social de nuestro territorio, más allá de hacerlo dentro de las entidades.

- Por otro lado, desde la experiencia iniciada en 2009, en la que se analizaron los servicios de las entidades en clave de participación, de la que ya hemos hablado (Grupo de Participación de EAPN Euskadi, 2011), las personas nos demandaban opinar sobre los servicios, ayudas y leyes que directamente les afectaban en el día a día.

El I Encuentro de Participación de EAPN Euskadi se celebró el 27 de junio de 2014, con presencia de más de un centenar de personas trabajadoras y beneficiarias de proyectos sociales (no sólo de las entidades que integran el Grupo de Participación), representantes de los órganos de dirección de entidades sociales y de la junta directiva de EAPN Euskadi, personal de la administración pública y de cargos políticos (ayuntamientos de Bilbao, San Sebastián y Vitoria-Gasteiz; diputaciones de Bizkaia y Gipuzkoa; Gobierno Vasco). Este encuentro sentó las bases de una dinámica que se mantendría en el tiempo y que se plasmaría en el nuevo plan estratégico de la Red para 2015-2017 (EAPN Euskadi, 2015a). Estableció también los siguientes objetivos, que la Red ha mantenido para todos sus procesos de participación:

- Dar voz, que sean las personas las que analicen las situaciones de pobreza y exclusión social que tienen lugar en Euskadi, con el fin de elaborar propuestas concretas de trabajo que se puedan hacer llegar a:

- Las entidades que forman parte de la Red, de las que forman parte estas personas.

- Las administraciones públicas que desarrollan e implementan políticas sociales.

- La sociedad en general.

- Elaborar documentos con propuestas concretas de políticas sociales, que la Red se compromete a hacer llegar a la Administración o a quien corresponda.

Los encuentros de participación establecen una dinámica de trabajo que tiene dos partes diferenciadas y que toman en cuenta todo lo que hemos ido señalando anteriormente:

- Primero, trabajamos en cada una de las entidades las cuestiones seleccionadas para el encuentro anual, y allí presentamos las conclusiones obtenidas en ese proceso. Para ello, se construyen guías metodológicas de apoyo a los y las profesionales, además de otros materiales, a fin de que cada entidad pueda seleccionar y adaptar las partes que desee. Se propone realizar, al menos, un taller en cada una de las entidades.

- Las personas que participan en los talleres de cada entidad deciden si quieren participar después en el encuentro general o no, siempre teniendo en cuenta el límite de plazas.

Esta dinámica implica, necesariamente, un trabajo previo por parte de los y las profesionales, no sólo de preparación de los talleres y del encuentro, sino de información y sensibilización a las personas atendidas en los servicios. No se trata, como hemos ido explicando, de obligar a participar, sino de proponer cauces y espacios de participación. Supone también una implicación por parte de los y las profesionales, que tienen que informar y trabajar con las personas la toma de decisiones, con el tiempo y los recursos que ello requiere. Por último, conlleva algo muy importante y necesario que señalábamos al principio del artículo, que es la apuesta clara y arriesgada de convertir la participación de las personas en situación o en riesgo de pobreza y exclusión social en una de las líneas estratégicas de la organización.

Desde 2014, EAPN Euskadi ha mantenido este compromiso promoviendo estos espacios (EAPN Euskadi, 2014, 2015b, 2016 y 2017b) y haciendo que las personas que participan en ellos tomen conciencia de que deben ser tenidas en cuenta en el diseño de las políticas sociales vascas. Asimismo, trabaja para que todas las propuestas de las personas que son resultado de los procesos de participación que se llevan a cabo en las entidades y en los encuentros sean elevadas a las administraciones públicas o adonde competa en cada caso. En este sentido, queremos señalar la comparecencia en el Parlamento Vasco de miembros de la junta directiva de la Red para presentar las conclusiones del Encuentro de 2016 y elevar, así, las propuestas de las personas en situación o riesgo de exclusión social al nuevo Plan Vasco de Inclusión Social (EAPN Euskadi, 2017a); como hemos dicho, sin medias tintas, sin ser la voz de las y los profesionales, sino constituyéndose como altavoz de las personas en situación y riesgo de exclusión social de Euskadi.

Cuando en EAPN Euskadi hablamos de participación, debemos hablar de derechos, de ciudadanía, de acción colectiva, de empoderamiento y de muchas más cosas. Debemos hablar de lo que nos afecta en el día a día y debemos enmarcarlo en las políticas que nos afectan en ese día a día. Como red de lucha contra la pobreza y la exclusión social en Euskadi, creemos que hablar de participación implica hablar de participación ciudadana, de participación comunitaria, de participación social, pero, sobre todo, de participación política, porque es este tipo de participación la que puede derivar en cambios significativos de las políticas sociales; cambios que se van a reflejar en la calidad de vida de las personas que atendemos en las entidades sociales. Todo este proceso de participación lo hacemos conociendo y dando a conocer la información, opinando y recogiendo las opiniones de las personas para que no vuelvan a ser olvidadas e invisibles (según sus 
propias palabras), y apoyando a las personas para que formen parte de los procesos de decisiones en torno al diseño de nuevas políticas sociales. Porque, parafraseando a Eduardo Galeano, "mucha gente pequeña, en lugares pequeños, haciendo cosas pequeñas, puede cambiar el mundo". 
ARTEIN, S. (1969): “A ladder of citizen participation”, Journal of the American Institute of Planners, vol. $35, \mathrm{n}$ 4, págs. 216-224.

CARRASQUILLA, M. C.; y CARNACEA, M. A. (2013): Crea, juega y participa. Caja de herramientas para la participación. Cuaderno conceptual, Madrid, EAPN España [<http://www.participacionsocial. org/cajaHerramientas/>].

EAPN ESPAÑA (2010): Guía metodológica de la participación social de las personas en situación de pobreza y exclusión social, Madrid, EAPN España [rhttp://www.eapn.es/ARCHIVO/documentos/ recursos/2/guia_participacion.pdfs].

EAPN EUSKADI (2017a): “EAPN Euskadi comparece en el Parlamento para llevar la voz de las personas en situación de vulnerabilidad y sus propuestas al nuevo Plan Vasco de Inclusión Social” [nota de prensa], EAPN Euskadi [rhttp:// www.eapneuskadi.org/es-pos_2\&nodo_ Id $=156 \&$ rel2 $=$ pos $>]$.

- (2017b): IV Encuentro de Participación de Euskadi: la Carta de Derechos Fundamentales de la U.E. [vídeo], EAPN Euskadi [«https://www.youtube. com/watch?v=31lwMMAPySU〉].

- (2016): III Encuentro de Participación de Euskadi: ¿qué queremos que sea el nuevo Plan de Inclusión Activa de Euskadi? [vídeo], EAPN Euskadi [<https://www.youtube.com/ watch?v=fGEZzPImLrM〉].

- (2015a): II Encuentro de Participación de Euskadi: las elecciones de mayo 2015 [vídeo], EAPN Euskadi ['https://youtu.be/d-XSPLDCYHg〉].

- (2015b): Plan Estratégico 2015-2017, EAPN Euskadi [<http://www.eapneuskadi.org/include/
uploads/files/PLAN\%20ESTRATEGICO $\% 20$ 2015\%202017\%20EAPN\%20EUSKADI.pdf〉].

- (2014): I Encuentro de Participación de Euskadi [vídeo], EAPN Euskadi [khttps://youtu.be/OEP7PZ83|w>].

GRUPO DE PARTICIPACIÓN DE EAPN EUSKADI (2011): La participación de las personas como herramienta para la inclusión social. Experiencias piloto en entidades sociales para el fomento de la participación, EAPN Euskadi [shttp://www.eapneuskadi.org/ include/uploads/nodo/Participacion-comoherramienta.pdf $\mathrm{f}$.

SUSÍAS, C. (2012): “Presentación”, en DE LA RIVA, F. (coord.), Foro de debate 2. Participación para la inclusión y la transformación social, Madrid, EAPN España, págs. 9-10 [rhttp://www. eapn.es/ARCHIVO/documentos/recursos/2/ Forodedebate2.definitivo.pdf>].

SUSO, A. (2012): “¿Qué formas de participación efectiva deben tener las personas excluidas o en riesgo de exclusión social en el desarrollo de sus propios procesos de inclusión, de los programas y proyectos que los articulan? ¿Cuáles son las principales dificultades y resistencias para la participación social efectiva de las personas excluidas o en riesgo de exclusión? ¿De qué formas podrían enfrentarse a superarse estas resistencias?", en DE LA RIVA, F. (coord.), Foro de debate 2. Participación para la inclusión y la transformación social, Madrid, EAPN España, págs. 71-74 [<http://www. eapn.es/ARCHIVO/documentos/recursos/2/ Forodedebate2.definitivo.pdf $>$ ]. 